\section{Vertebrate embryo handedness}

SiR - There has recently been renewed discussion of the various more or less consistent left-right asymmetries, that is the handedness, of the vertebrate body (see ref. 1). A strong but transient handedness is consistently visible in the bird embryo at stages well before formation of any of the organ systems. The chick Hensen's node, a structure where dorsal axial tissue for the future body is being fed into position in a head-to-tail sequence, assumes a 'handed' structure for just two to three hours. The tissue leaving the node at this time will form the mid- to hindbrain regions of the future head, where obscure asymmetries of structure in nervous system and pharynx roof are found, especially in the vertebrate relatives the protochordates $^{2}$. When three or four somites have segmented in the chick, apparent symmetry is regained, to last for many hours until appearance of the first permanent but localized handedness, that of the heart. The node handedness has been recorded by others, notably Lepori ${ }^{3}$ for the duck blastoderm, but information about its stability to developmental disturbance or relationship with subsequent handed phenomena is lacking.

During the asymmetry, the front end of the primitive streak is diverted towards the left as viewed from above, while the anteriorly emerging notochordal rod the vertebrate midline structure - has its root at the right, before bending to adopt a course that extends the overall line of the streak or even swings rightwards.
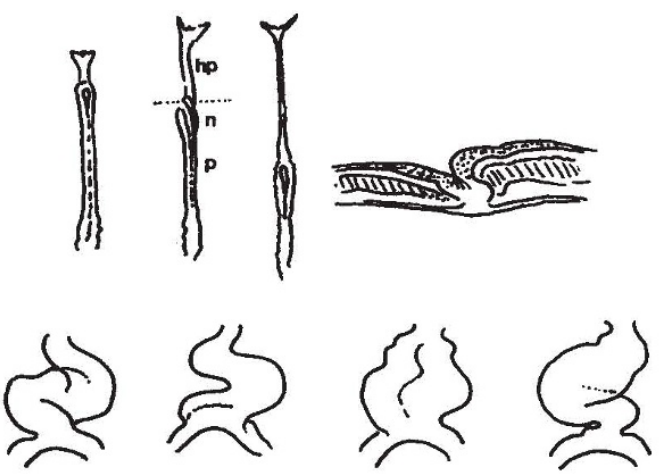

Sketches show the following: upper row from left to right first shows the appearance of chick gastrulation from dorsal aspect, at early stage 5 , stage 6 and early stage 8 (ref. 5). The node $(n)$ regresses to shorten the primitive streak ( $p$ ) and extend the head process (hp). Only when the fan-shaped prechordal tissue is already in place, but just the anteriormost notochord region has left the node, is pronounced asymmetry seen. Sketch at extreme right shows a typical transverse section tracing through the asymmetrical node, orientated as if an observer were facing anteriorly. Asymmetries are obvious in node tissue, in flanking mesoderm (the middle layer) and in overlying midbrain-level neural plate. The lower row shows in dorsal view, from left to right, two versions of the normally handed heart tube at the 12 somite stage, one with unclear handedness, and one with reverse handedness.
There are also marked differences in thickness and form of the neural plate and the sheet of emerging mesoderm on either side at the node (see figure). I have observed no instance of reversal or absence of this appearance in almost 150 blastoderms dissected from the egg at these stages, and it is independent of mechanical tensions that normally stretch the blastoderm, since it is maintained for ours in free-floating, living specimens.

The occurrence of individual bodies with various unusual combinations of left-right asymmetry in the developed organ systems (for example, heart, viscera, human brain function) may indicate that variously reliable developmental mechanisms derive each visible handedness from a hidden primary one, which underlies the consistent asymmetry normally found ${ }^{1}$. Ring culture of chick blastoderms tretched on the inverted vitelline membrane ${ }^{4}$, from stages before and during node asymmetry, has consistently resulted in around $7 \%$ reversal of heart handedness. I have therefore cultured blastoderms from primitive-streak stages before the definitive node has developed, scored individuals for node handedness when this tage is reached, and ultimately examined he same individuals for heart handedness.

Normal node handedness was exhibited by every one of 75 such blastoderms at head-process stages, even though these were reached in a delayed way 7-12 hours fter onset of culture, and the whole node egion was often miniaturized and contained abnormally few cells. However, when examined at the 13-somite stage a further 20 hours later, 6 embryos had clear situs inversus of the heart, whereas a further 7 had hearts of abnormal symmetry or unclear handedness (see figure). Early gastrular handedness thus seems a more stably developing aspect of the bird embryo's structure than that of at least one later organ, the heart. Heart situs is in fact most liable to perturbation when embryos are placed in culture late, at times between the resymmetrization of the node and actual heart development. It can thus be determined subsequent to, as well as potentially independently of, node handedness.

Viewed as if it were a primitive adult organism, the chick embryo rudiment at its most strongly handed stage would not be considered as primarily bilaterally symmetrical. Certain fossil evidence suggests that the chordate phylum evolved from a truly bilaterally symmetrical organism that 'fell over' on its original right-hand side, to re-acquire a new more superficial left-right symmetry by progressive modification of the former dorsal (new right) edge of the body. One might indeed fantasize that in this very robust present-day handed structure, the bird gastrula displays its origins via dexiothetism $^{1}$, the application of a former right side to the substrate. Transient gastrular handedness can be easily observed in the translucent bird blastoderm, but should be actively searched for in other vertebrate types.

\section{Jonathan Cooke}

National Institute for Medical Research, The Ridgeway, Mill Hill,

London NW7 1AA, UK

$$
\begin{aligned}
& \text { 1. Jefferies, R. CIBA Fdn Symp. 162, 94-127 (1991). } \\
& \text { 2. Young, J. Z. The Life of Vertebrates } 2 \text { nd edn (Oxford } \\
& \text { University Press, 1962). } \\
& \text { 3. Lepori, N. G. Monitore Zool., Ital. 3, 33-53 (1969). } \\
& \text { 4. New, D. A. T. J. Embryol. exp. Morph. 3, 320-331 } \\
& \text { (1955). } \\
& \text { 5. Hamburger, V. \& Hamilton, H. L. J. Morph. 88, 49-92 } \\
& \text { (1951). }
\end{aligned}
$$

\section{$\mathrm{Pi}$ in the sky}

SIR - The notion that at root "all is number" is a belief that can be traced back at least as far as the Pythagoreans, in around $500 \mathrm{BC}^{1}$. Although our current scientific conception of the Universe is indeed mathematical, it is hardly of the unalloyed simplicity conceived by the Ancient Greeks. But the mere appearance of the night sky can be used to deduce a remarkably accurate value for the transcendental number $\pi$.

This surprising result follows from an application of theorems in analytical number theory. For any integer $p$, the number of smaller integers with which $p$ shares no factors is $\phi(p)$, where $\phi$ is the Euler totient function ${ }^{2}$. Now, let $(p, q)$ be a pair of integers chosen at random such that $q>0,1 \leq p \leq q \leq n$, where $n$ is large. There are of the order of $n^{2} / 2$ pairs of such integers, and the number of them whose members are relatively prime is $\Phi(n)=\phi(1)+\phi(2)+\ldots+\phi(n)$. The probability that two random integers are relatively prime is thus $2 \Phi(n) / n^{2}$, where for large $n$ it can be shown that ${ }^{2}$

$$
\Phi(n) \approx n^{2} / 2 \zeta(2)
$$

with $\zeta(2)=\pi^{2} / 6$, the value of the Riemann zeta-function for reciprocal squares. Thus the probability that two integers chosen at random are relatively prime is

$$
P(p, q)=6 / \pi^{2}
$$

This result enables us to calculate a value of $\pi$ from a large random sample of integers: we form pairs of such integers, and determine what proportion are relatively prime.

The scattering of the brightest stars over the night sky sugests they may allow a 'celestial' estimate of $\pi$. Let $\left(\alpha_{i}, \delta_{i}\right)$ be the right ascension and declination of the $i$ th star in the night sky. Its angular distance from the $j$ th star is then $\Delta_{i j}$, where ${ }^{3}$ 